Cahiers $d u$ MONDE RUSSE

\section{Cahiers du monde russe}

Russie - Empire russe - Union soviétique et États indépendants

$46 / 1-2 \mid 2005$

La Russie vers 1550

\title{
Les réformes du milieu du xvie siècle et l'évolution structurelle de la noblesse russe
}

Andrej P. PAVLOV

\section{OpenEdition}

\section{Journals}

Édition électronique

URL : https://journals.openedition.org/monderusse/8783

DOI : $10.4000 /$ monderusse. 8783

ISSN : $1777-5388$

\section{Éditeur}

Éditions de l'EHESS

\section{Édition imprimée}

Date de publication : 1 janvier 2005

Pagination : 91-106

ISBN : 2-7132-2055-6

ISSN : $1252-6576$

Référence électronique

Andrej P. PAVLOV, «Les réformes du milieu du xvie siècle et l'évolution structurelle de la noblesse russe », Cahiers du monde russe [En ligne], 46/1-2 | 2005, mis en ligne le 01 janvier 2007, consulté le 03 septembre 2022. URL : http://journals.openedition.org/monderusse/8783; DOI : https://doi.org/ 10.4000/monderusse. 8783 
chercher : repérer : avancer

Cet article est disponible en ligne à l'adresse :

http://www.cairn.info/article.php?ID REVUE=CMR\&ID NUMPUBLIE=CMR 461\&ID ARTICLE=CMR 4610091

\title{
Les réformes du milieu du xvie siècle et l'évolution structurelle de la noblesse russe
}

\author{
par Andrej P. PAVLOV
}

\section{Editions de l'EHESS | Cahiers du monde russe}

\author{
2005/1-2 - Vol 46 \\ ISSN 1252-6576 | ISBN 2713220556 | pages 91 à 106
}

Pour citer cet article :

-P. PAVLOV A., Les réformes du milieu du xvie siècle et l'évolution structurelle de la noblesse russe, Cahiers du monde russe 2005/ 1-2, Vol 46, p. 91-106.

Distribution électronique Cairn pour les Editions de l'EHESS.

(C) Editions de l'EHESS. Tous droits réservés pour tous pays.

La reproduction ou représentation de cet article, notamment par photocopie, n'est autorisée que dans les limites des conditions générales d'utilisation du site ou, le cas échéant, des conditions générales de la licence souscrite par votre établissement. Toute autre reproduction ou représentation, en tout ou partie, sous quelque forme et de quelque manière que ce soit, est interdite sauf accord préalable et écrit de l'éditeur, en dehors des cas prévus par la législation en vigueur en France. Il est précisé que son stockage dans une base de données est également interdit. 


\section{LES RÉFORMES DU MILIEU DU XVIe SIÈCLE ET L'ÉVOLUTION STRUCTURELLE DE LA NOBLESSE RUSSE}

L'unification politique des pays russes de la fin du XVe au début du XVI siècle contribue largement au processus de formation d'un corps unique de serviteurs du prince et à la définition du statut de l'élite dirigeante, à savoir les membres de la cour du tsar.

Au fur et à mesure que l'unification progresse, que le nouvel État doit résoudre, à l'intérieur comme à l'extérieur, des problèmes devenus plus complexes, la seconde moitié du XVe et le premier tiers du XVI ${ }^{e}$ siècle voient crôtre rapidement le nombre des serviteurs du prince [služilye ljudi]. Si l'on examine les généalogies déposées au Secrétariat de la Guerre par les représentants des familles de serviteurs du souverain à la fin du XVII siècle $^{1}$, on constate que, dans la majorité des cas, les légendes des clans font remonter l'entrée de leurs ancêtres au service des souverains russes aux règnes de Basile II, Ivan III et Basile III.

L'augmentation du nombre de serviteurs du prince rend inévitable un processus de parcellement des patrimoines [sing. votčina]. La Russie ne connaît pas, avant Pierre le Grand, le principe de l'héritier unique, ou majorat. Les patrimoines étaient divisés également entre les fils du propriétaire terrien, ce qui entraînait fréquemment la disparition du patrimoine familial et la déchéance de maisons entières de serviteurs du prince. La crise du patrimoine est aggravée par le transfert fréquent de patrimoines laïques aux monastères, sous forme de franche aumône [vklad]. La création d'une nouvelle forme de propriété foncière, le bénéfice [pomest'e], aida à surmonter la crise. La concession en précaire des terres confisquées aux bojare de Novgorod à un groupe important de serviteurs moscovites fut un grand pas en avant vers la systématisation du bénéfice. Celui-ci fut étendu progressivement à l'ensemble du territoire et devint le type prépondérant de propriété foncière laïque.

1. RGADA, fonds 217 , inventaire $n^{\circ} 18$. 
Les conséquences sociales et politiques de la réforme furent d'une grande portée, son résultat ultime étant la transformation, au XVI ${ }^{e}$ siècle, de l'ancienne aristocratie des bojare de l'époque du morcellement, en un corps de «gentilshommes », ou serviteurs militaires du prince ${ }^{2}$.

La centralisation du pays eut pour corollaire l'abolition du «service libre » (vassalité), ou de ce qui en restait, et son remplacement par des rapports de sujétion inédits jusqu'alors. Ce processus, que l'on observe également dans d'autres pays européens, se distingue en Russie par des traits spécifiques. L'unification politique s'y accompagne de mesures relativement brutales d'uniformisation des structures socio-politiques sur l'ensemble du territoire, et de la liquidation résolue des anciens droits et privilèges. Ainsi, dans les pays (Novgorod, Pskov, Smolensk, Sévérie) où les institutions socio-politiques et la structure de la propriété foncière différaient de celles de Moscou, les bojare, principaux propriétaires locaux, furent tous expulsés de leurs domaines, transformés en bénéfices pour le compte des serviteurs du prince, venus de différentes « villes » $d u$ « pays de Moscou ». L'incorporation des pays et des principautés de la Russie du Nord-Est n'entraîna pas, il est vrai, de refonte radicale des structures existantes de la propriété foncière, ni de déplacements massifs des propriétaires vers d'autres districts du pays. Cependant, même dans ces régions, la solidarité corporative qui unissait anciennement les bojare locaux fut également détruite dans une large mesure ${ }^{3}$.

Le processus d'unification et de centralisation eut pour effet de priver les bojare, les bojare cadets et les simples gentilshommes de leur droit de désaveu [ot"ezd], qui leur permettait de passer au service d'un autre souverain. Le désaveu est désormais perçu comme acte de trahison. À partir de la fin du Xve siècle apparaissent des documents [poručnye zapisi] dont les signataires se portent garants des bojare soupçonnés de vouloir désavouer le grand-prince, et donc de le trahir ${ }^{4}$.

Les serviteurs du prince perdent non seulement leur liberté politique, mais aussi leur autonomie économique par rapport à l'État. Ainsi, à la charnière entre XV et $\mathrm{XVI}^{\mathrm{e}}$ siècle, des propriétaires fonciers laïques de rangs très variables (depuis les détenteurs de patrimoines importants jusqu'aux petits bénéficiers [sing. pomeščik]) se voient retirer leurs privilèges fiscaux ${ }^{5}$.

2. V. B. Kobrin, Vlast' i sobstvennost' v srednevekovoj Rossii (XV-XVI VV.) [Pouvoir et propriété en Russie médiévale, $X V$-XVI $\mathbb{I}^{\mathrm{e}}$ siècles], Moscou, 1985, p. 133 ; R. G. Skrynnikov, Tragedija Novgoroda [La tragédie de Novgorod], Moscou, 1994, p. 26-27.

3. B. N. Florja, «O putjah političeskoj centralizacii Russkogo gosudarstva (na primere Tverskoj zemli)» [« Voies et moyens de la centralisation politique en Russie (sur l'exemple du pays de Tver') »], in Obščestvo i gosudarstvo feodal'noj Rossii [État et société en Russie féodale], Moscou, 1975, p. 281-290.

4. S. M. Kaštanov, «Gosudar’ i poddannye na Rusi v XIV-XVI vv. » [« Le souverain et ses sujets en Russie aux XIV-XVI siècles »], in In memoriam. Sbornik pamjati Ja. S. Lur'e [Hommages à Ja. S. Lur’e], Saint-Pétersbourg, 1997, p. 226.

5. B. N. Florja, « Podatnye privilegii svetskih feodalov v period obrazovanija edinogo Russkogo gosudarstva » [ « Les privilèges fiscaux des seigneurs laïques au cours de l'unification de la Russie»], in Rossija XV-XVIII stoletij. Sbornik naučnyh statej [La Russie aux XV$X V_{I I I}{ }^{e}$ siècles. Recueil d'articles], Volgograd - Saint-Pétersbourg, 2001, p. 28-37. 
Au cours de la seconde moitié du XVe et du premier tiers du XVI e siècle, la possession foncière des princes change, elle aussi, de caractère. Les droits souverains particuliers qu'ils exerçaient sur leur territoire sont graduellement abolis et leurs domaines lignagers se rapprochent de plus en plus des patrimoines ordinaires des bojare $^{6}$. Le nombre croissant de bénéfices rend familière l'idée selon laquelle les propriétaires de terres patrimoniales doivent au grand-prince le service militaire au même titre que les bénéficiers.

Tous ces facteurs réunis ont marqué le processus de formation d'un corps unique de la noblesse, dans la mesure où celui-ci se présente sous la forme d'un corps de serviteurs, dont tous les membres (y compris l'aristocratie princière ou les bojare) dépendent étroitement de la monarchie.

À la charnière entre $\mathrm{XV}^{\mathrm{e}}$ et $\mathrm{XVI} \mathrm{I}^{\mathrm{e}}$ siècle, on voit apparaître une cour unifiée du tsar, qui se constitue progressivement pendant que l'on procède à une refonte de ses structures. La première étape concerne les grades de membres du Conseil (bojare et quartiers-maîtres) et les dignités auliques. Dans les listes du premier tiers du $\mathrm{XVI}^{\mathrm{e}}$ siècle les grades clairement définis sont: les bojare [singulier : bojarin], les quartiers-maîtres [sing. okol'ničij], le connétable [konjušij], les maîtres de l'Hôtel [sing. dvoreckij], le trésorier [kaznačej], le garde du Sceau [pečatnik], le maître de l'Armurerie [oružničij], les cubiculaires [sing. postel'ničij], les grands fauconniers [sing. sokol'ničij], les maîtres de l'Écurie [sing. jasel'ničij], les grands veneurs [sing. lovčij]. Simultanément, le Conseil [bojarskaja duma] s'affirme comme organe suprême du pouvoir d'État, auquel il revient d'examiner l'ensemble des problèmes importants à l'échelle du pays. Mihail Krom a démontré de manière convaincante que l'étape décisive dans ce processus fut la crise politique des années 1530-1540, lorsque, du fait de la minorité d'Ivan IV, le Conseil tout entier (et non point des fractions isolées) prend en main le gouvernement du pays. C'est au début des années 1540 que remontent les premières références à des arrêts du Conseil [bojarskij prigovor]. Enfin, c'est pendant la période dite « du gouvernement des bojare » que l'on trouve attestée, pour la première fois, la participation des représentants de tous les grades du Conseil à la prise de décisions gouvernementales ${ }^{7}$.

À l'instar des grades du Conseil, d'autres grades de la cour prennent forme dans la première moitié du XVI ${ }^{\mathrm{e}}$ siècle. Il s'agit des panetiers [sing. stol'nik], des gentilshommes de la chambre [sing. strjapčij] et, probablement, des pages [sing. žilec] (les premiers renseignements explicites concernant ces deniers datent de 1552, mais déjà, dans la première moitié du siècle, on parle des «adjoints aux gardes du corps » [poddatnej u rynd], fonction de page par excellence). Vers le milieu du

6. V. B. Kobrin, op. cit. (note 2), p. 48-92.

7. M. M. Krom, «"Mne sirotstvujušču, a carstvu vdovstvujušču": krizis vlasti i mehanizm prinjatija rešenij v period bojarskogo pravlenija (1530-1550) » [ " "Je suis orphelin et l'État veuf" : la crise du pouvoir et le mécanisme de prise de décision pendant le "gouvernement des bojare" (années 1530-1550) »], in Rossijskaja monarhija : voprosy istorii i teorii. Mežvuzovskij sbornik statej, posvjaščennyh 450-letiju učreždenija carstva v Rossii (1547-1997 gg.) [ La monarchie russe : questions d'histoire et de théorie. Recueil interuniversitaire d'articles consacrés au 450 anniversaire de l'institution impériale en Russie (1547-1997)], Voronež, 1998, p. $40-49$. 
siècle, la cour du tsar se présente déjà comme une structure socio-politique aux contours bien définis. Grâce aux recherches de Vladislav Nazarov, nous savons que, dès la première moitié du XvI ${ }^{\mathrm{e}}$ siècle, la composition du personnel de la cour fait l'objet de documents spéciaux - les rôles de la cour [sing. bojarskij spisok].

Les fragments de rôles datant des années 1546-1547 qu'a découverts Vladislav Nazarov donnent un tableau suffisamment clair et complet de la structure de la cour à cette époque. Elle était composée des groupes suivants : d'une part, les grades du Conseil et les officiers de la Couronne (bojarin, quartier-maître, connétable, maître de l'Hôtel, trésorier, maître de l'Armurerie, cubiculaire, garde du Sceau, grand fauconnier, grand veneur), déjà mentionnés ci-dessus ; d'autre part, les panetiers et gentilshommes de la chambre, ainsi que les princes [sing. knjaz'] et les gentilshommes de la cour [sing. dvorovoj syn bojarskij plur. deti bojarskie], qui figurent soit dans des rôles particuliers, sous le nom de différents princes (de Suzdal', d'Obolensk, de Jaroslavl', etc.), soit dans des rôles de "villes », c'est-à-dire de corporations urbaines (Tarusa, Moscou, Tver', Volok, Vjaz'ma, etc.) ${ }^{8}$. La première moitié du XVI ${ }^{\mathrm{e}}$ siècle est à cet égard une période décisive, où prend forme la hiérarchie des grades de la cour. Plus que le grade, toutefois, c'est l'appartenance territoriale qui préside à l'organisation de la cour à cette époque. Des panetiers, des gentilshommes de la chambre, des princes et des gentilshommes de la cour qui figurent dans les rôles de la cour des années 1540 sont à la fois membres de la cour et partie intégrante des corporations locales de serviteurs du souverain. Leurs noms nous sont connus non seulement par les rôles de la cour, mais aussi par les documents qui recensaient la noblesse provinciale. Fait caractéristique, les noms de nombreux panetiers et gentilshommes de la chambre, répertoriés dans les rôles de la cour de 1546-1547, figurent également dans le document dit Cahier de la cour [Dvorovaja tetrad'] des années 1550, mais cette fois sous la rubrique de leurs districts respectifs en qualité de représentants de la noblesse locale.

La prédominance des structures territoriales à la cour du tsar est étroitement liée aux particularités de l'intégration des élites locales à la noblesse moscovite au cours de l'unification du pays. Ainsi, les anciens vassaux des principautés nouvellement rattachées à Moscou (probablement pour éviter d'embrouiller les rapports hiérarchiques de préséance entre les serviteurs du grand-prince) furent inclus dans la cour unifiée du souverain et inscrits dans les rôles correspondants par groupes entiers selon leur appartenance territoriale, en tant que représentants de leurs corporations locales. Boris Nikolaevič Florja a étudié les formes et les circonstances qui accompagnèrent l'intégration de l'aristocratie locale dans la cour unifiée, sur l'exemple du pays de Tver' 9 . Parallèlement, l'aristocratie moscovite et les membres de la cour du grand-prince ont pratiqué, entre le début du XIV siècle et le premier tiers du $\mathrm{XVI}^{\mathrm{e}}$ siècle, une politique d'acquisitions foncières massives sur le territoire des pays

8. V. D. Nazarov, «O strukture gosudareva dvora v seredine XVI v. » (« La structure de la cour du souverain au milieu du XVI ${ }^{\mathrm{e}}$ siècle »), in Obščestvo i gosudarstvo feodal'noj Rossii, op. cit. (note 3), p. 40-54.

9. B. N. Florja, « O putjah... », op. cit. (note 3), p. 281-290. 
et des principautés tombés dans le giron de Moscou ${ }^{10}$. Ces mutations foncières entraînaient l'enracinement des serviteurs moscovites en province. Liés aux propriétaires locaux par leurs intérêts fonciers, leurs rapports de parenté et autres, ils s'intégraient d'autant plus facilement aux communautés nobles de l'endroit. Le Cahier de la cour permet de suivre les différentes façons dont se construisent les groupes nobiliaires régionaux. Ainsi, les représentants à la cour de la corporation des serviteurs de Tver' sont divisés en deux rubriques, dont l'une s'intitule « Tver' » et l'autre, « Bénéficiers de Tver'».

Cependant, la structure hiérarchique des grades de la cour restait à bien des égards inachevée. Du fait de l'incorporation de nouveaux territoires, la cour des princes moscovites s'agrandit considérablement quant aux effectifs, mais elle y perdit en mobilité. Une part importante des membres de la cour (en particulier les serviteurs venant de régions nouvellement rattachées à Moscou) ne possédaient ni bénéfices ni patrimoines à proximité de la capitale, ce qui entraînait de sérieux inconvénients lorsqu'ils étaient de service à Moscou ${ }^{11}$. Bon nombre de gentilshommes de la cour s'installèrent dans les «villes » de province et ne furent pour ainsi dire plus jamais convoqués à la capitale pour servir à la cour. Ni la composition, ni la structure hiérarchique de celle-ci n'étaient encore du reste clairement définies. L'engagement insuffisant de la noblesse régionale dans le service moscovite se manifesta d'une manière flagrante lors de la période de crise des années 1530-1540 (le «gouvernement des bojare»). On vit alors les contingents nobiliaires de province changer de camp du jour au lendemain et se ranger alternativement derrière telle ou telle fraction de l'aristocratie.

Le besoin se faisait sentir d'une remise en ordre de la cour, tant du point de vue de ses effectifs que de sa structure hiérarchique, afin de créer un dispositif permettant de rendre plus efficace le service des membres de l'élite. Ce fut l'objectif de la réforme de la cour du milieu du XVI ${ }^{\mathrm{e}}$ siècle, dont l'élément essentiel fut la réforme dite « du Millier » (1550). Son contenu est assez clairement résumé dans un édit d'octobre 1550, qui prévoit la concession en précaire de terres dans la région de Moscou à un millier des «meilleurs serviteurs ». Ces derniers (choisis parmi les gentilshommes de la cour et en partie parmi les gentilshommes des «villes» [sing. gorodovoj syn bojarskij]) se voient attribuer des bénéfices dans le district de Moscou et quelques districts adjacents (Dimitrov, Ruza, Zvenigorod), de manière à ce qu'ils soient toujours sous la main et prêts à " partir en mission ", c'est-à-dire à exécuter les requêtes gouvernementales. La taille des bénéfices dépend du rang du serviteur (de son grade, de son lignage, de son degré d'intimité avec le souverain). Ainsi s'explique la structure du Registre du Millier [Tysjačnaja kniga]. La liste des noms commence par les rôles des membres du Conseil et des courtisans les plus haut placés (bojare,

10. Au sujet des acquisitions foncières des serviteurs moscovites du prince dans le pays de Rostov à partir du XIVe siècle, voir S. V. Strel'nikov, «Zemlevladenie v Rostovskom uezde v XIV - pervoj treti XVII vv. » [Propriété foncière dans le district de Rostov, XIVe- premier tiers du XVII ${ }^{\mathrm{e}}$ siècle], résumé de thèse, Saint-Pétersbourg, 2003.

11. S. B. Veselovskij, Issledovanija po istorii opričniny [Études d'histoire de l'opričnina], Moscou, 1963,p. 78-79. 
quartiers-maîtres, maître de l'Armurerie, trésoriers), auxquels est attribuée une dotation foncière de 200 arpents [sing. četvert] près de Moscou. Les autres bénéficiaires de la réforme (princes et gentilshommes) sont divisés en trois sections, ou classes, dont chacune à son tour comprend des listes princières («princes d'Obolensk », « princes de Jaroslavl`», etc.), et des rubriques territoriales (Perejaslavl’, Kolomna, Tver', etc.). Les bénéficiaires appartenant à la première classe se voient octroyer, comme les membres du Conseil, une dotation de 200 arpents. Ceux qui relèvent de la deuxième classe ont droit à 150 et ceux de la troisième classe (les plus nombreux), à 100 arpents. Ces «meilleurs serviteurs » étaient censés, selon l'édit, constituer le noyau de la nouvelle cour, dont les effectifs étaient mis en accord avec les besoins réels de l'administration du pays. Cependant, le Registre du Millier de 1550 n'est pas un rôle des membres de la cour à proprement parler, et la liste est loin d'être exhaustive. N'y sont recensés que ceux parmi les serviteurs qui ont droit à un bénéfice dans le district de Moscou, ce qui en exclut, aux termes de l'édit de 1550, les propriétaires de terres patrimoniales proches de la capitale.

Outre le Registre du Millier, un autre document du milieu du XVI e siècle, dit le Cahier de la cour, nous est parvenu ; il date, comme l'a démontré Aleksandr Zimin, de $1551 / 52^{12}$. Il répertorie un nombre de serviteurs autrement important que le Registre. Ce dernier ne dépasse que de peu mille personnes (dont près d'un tiers de nobles des régions de Pskov et de Novgorod), tandis que le Cahier énumère environ 3000 membres de la cour, résidant tous dans des villes du pays de Moscou (à l'exclusion de Novgorod et de Pskov). Il est communément admis que le Cahier constitue la liste exhaustive des membres de la cour (il a été utilisé comme document de référence pendant une décennie environ, du début des années 1550 au début des années 1560), tandis que les noms du Registre seraient ceux des « meilleurs serviteurs », de la partie « choisie» de la cour ${ }^{13}$. Toutefois, les recherches de Vladislav Nazarov ont montré que le Cahier de la courétait un document complexe, compilé à partir de matériaux de dates différentes, dont certains remontent au début des années $1540^{14}$. Il faut souligner également les singularités du texte : les serviteurs sont inscrits, le plus souvent, par clans entiers (mélangeant sur une même ligne les noms des frères aînés et cadets, des pères et des fils, des oncles et des neveux, etc.). Qui plus est, comme nous avons pu nous en assurer, on voit que parmi les membres recensés figurent également des individus décédés avant 1551/52, date de la composition du texte originel, ou encore des mineurs qui n'ont pas l'âge requis pour servir ${ }^{15}$. Le Cahier ne peut donc guère être

12. A. A. Zimin, éd., Tysjačnaja kniga 1550 g. i Dvorovaja tetrad’ 50-h godov XVI veka [Le Registre du Millier de 1550 et le Cahier de la cour des années 1550], Moscou - Leningrad, 1950 , p. 17.

13. A. A. Zimin, Reformy Ivana Groznogo [Les réformes d'Ivan le Terrible], Moscou, 1960, p. 371 ; S. B. Veselovskij, op. cit. (note 11), p. 81.

14. V. D. Nazarov, «K istočnikovedeniju Dvorovoj tetradi » [« Sur les sources du Cahier de la cour »], in Rossija na putjah centralizacii [La Russie en voie de centralisation], Moscou, 1982, p. 166-175.

15. A. P. Pavlov, Gosudarev dvor i političeskaja bor'ba pri Borise Godunove (1584-1605) [La cour du tsar et la lutte politique sous Boris Godunov (1584-1605)], Saint-Pétersbourg, 1992, p. 87-89. 
considéré comme un document reflétant la constitution réelle de la cour. Il est probable que la composition du Cahier a été motivée par la mise en pratique de la réforme « du Millier », afin de disposer du maximum de renseignements sur l'élite des serviteurs du tsar.

Peut-on, dans ces conditions, chiffrer les effectifs de la cour et déterminer sa composition? En premier lieu, sans aucun doute, il faut y inclure l'élite des serviteurs - les bénéficiaires de la réforme « du Millier». Mais on se souvient que le Registre du Millier de 1550 n'est pas complet, puisqu'il ignore les membres de la cour déjà pourvus de domaines aux environs de la capitale. C'est ainsi, très probablement, que s'explique l'absence de plusieurs membres du Conseil (18 bojare sur 32, 7 quartiers-maîtres sur 12 seulement sont inscrits dans le Registre de 155016). De fait, les noms de certains de ces absents se retrouvent dans les cadastres [sing. piscovaja kniga] du XVI ${ }^{\mathrm{e}}$ siècle pour le district de Moscou. Cependant, si une bonne part de l'élite a réussi à se procurer des terres près de Moscou, la grande majorité des membres de la cour en étaient apparemment dépourvus. Il est difficile dans ces conditions d'estimer le nombre de serviteurs du prince qui possédaient déjà un patrimoine près de la capitale au moment de la promulgation de l'édit de 1550 . On peut admettre toutefois que les serviteurs dans ce cas étaient relativement peu nombreux, et que c'était le Millier choisi qui constituait le noyau de la cour. Cette hypothèse est corroborée par les faits suivants : parmi les 250 capitaines et adjoints dont les noms figurent dans les registres des rangs [sing. razrjadnaja kniga] de la période 15491552, une vingtaine seulement n'est pas répertoriée dans le Registre ${ }^{17}$. Il comprend également la quasi-totalité (15) des panetiers qui apparaissent dans le rôle ${ }^{18}$ [ici : rospis ] de la campagne de Kazan' (1549-1550). Parmi les 12 pages (adjoints aux gardes du corps) qui participent à la campagne, un seul - Théodore Grigor'evič Sovin l'Aîné - ne figure pas dans le Registre. Vladislav Nazarov constate que 26 des 29 panetiers qui figurent dans le rôle de la cour de 1546 sont présents dans le Registre $d u$ Millier $^{19}$. On peut donc en conclure que la nouvelle cour prend son essor avec la réforme « du Millier », et que, inversement, la majorité des membres de cette cour se retrouvent parmi les bénéficiaires de la réforme.

En confrontant le Registre du Millier avec le Cahier de la cour (du moins les éléments comparables, à savoir la liste des gentilshommes des villes du pays de Moscou, dont 750 environ sont recensés par le Registre), on constate que la majorité (près de $85 \%$ d'entre eux) figurent également dans le Cahier. Cela permet de mieux comprendre les critères qui présidaient au choix des membres de la cour (des «meilleurs serviteurs ») parmi les représentants des élites nobiliaires locales. Premièrement, étaient exclus d'emblée ceux qui, en raison leur âge ou pour cause

16. A. A. Zimin, « Sostav bojarskoj dumy v XV-XVI vekah » [« La composition du Conseil aux XVe-XVIe siècles »], in AE 1957, Moscou, 1958, p. 63-72.

17. Razrjadnaja kniga, 1475-1605 [Le registre des rangs 1475-1605], Moscou, 1977, vol. I, fascicule II, p. 377, 379, 380 .

18. Ailleurs, « rôle » traduit le russe spisok $(\mathrm{NdT})$.

19. V. D. Nazarov, « O strukture... », op. cit. (note 8), p. 49. 
de maladie, étaient inaptes au service de cour. Le Registre du Millier omet fréquemment les noms qui, dans le Cahier de la cour, sont annotés « vieux » ou « malade », ainsi que les représentants des jeunes générations. Une autre catégorie éliminée à la sélection comprend ceux des gentilshommes de province qui, vers 1550, n'étaient plus convoqués (ou convoqués très rarement) pour servir à la cour de Moscou. Enfin, la notion même de «meilleur serviteur » impliquait que soient invités à la cour les représentants des lignages les plus anciens et les plus prestigieux par leurs états de service. De fait, le pourcentage de rejetons de familles connues, princières ou celles des boyards de haute naissance, est sensiblement plus élevé dans le Registre du Millier que dans le Cahier de la cour. Voilà qui est incompatible avec la théorie d'Ivan Smirnov et d'Aleksandr Zimin (dans un de ses premiers travaux), qui considérait la réforme « du Millier » comme favorable à la noblesse moyenne, et visant à évincer du gouvernement l'aristocratie des bojare ${ }^{20}$.

Les chercheurs ont déjà attiré l'attention sur le fait que la division des «meilleurs serviteurs» en trois classes, suivant l'importance de la dotation octroyée, ne devait rien au hasard et reflétait la structure hiérarchique de la cour. Cela apparaît clairement dans le Registre, où les deux premières classes correspondent à l'élite de la cour, tandis que la troisième regroupe au contraire la plus basse catégorie. La répartition numérique des « meilleurs serviteurs » est révélatrice à cet égard. La première classe comprend 33 personnes, la deuxième - 79, mais la troisième en compte 614. On ne trouve que sept gentilshommes de Novgorod dans la première classe, alors que 317 d'entre eux sont inscrits dans la deuxième.

Il suffit de jeter un coup d'œil sur les listes du Registre du Millier pour constater que, dans les deux premières classes, une nette majorité des inscrits descendent de maisons de princes ou de bojare. En revanche, les serviteurs de la troisième classe (pour les Moscovites) et de la deuxième (pour les Novgorodiens) appartiennent pour la plupart à des familles nobles ordinaires. Cependant, le classement des membres du Millier choisi ne coïncide pas totalement avec leur rang en matière de préséances. Ainsi, les gardes du corps [sing. rynda] de la campagne de Kazan' (1549/50) sont énumérés dans l'ordre suivant par le registre des rangs :

\author{
Prince Georges Ivanovič Šemjakin-Pronskij (1 $1^{\text {re }}$ classe) \\ Prince André Ivanovič Tatev ( $3^{\mathrm{e}}$ classe) \\ Basile Andreevič Buturlin ( $3^{\mathrm{e}}$ classe) \\ Prince Michel Petrovič Repnin ( $2^{\mathrm{e}}$ classe $)$ \\ Prince Pierre Ivanovič Tatev ( $2^{\mathrm{e}}$ classe $)$
}

On peut remarquer également que le classement des panetiers ne correspond pas non plus à la hiérarchie des grades de la cour. Parmi les panetiers de la campagne de Kazan' (1549/50), nous trouvons des personnages appartenant indifféremment aux

20. A. A.Zimin, Reformy..., op. cit. (note 13), p. 266 ; I. I. Smirnov, Očerki političeskoj istorii Russkogo gosudarstva 30-50-h gg. XVI veka [Essais d'histoire politique de la Russie des années 1530-1550], Moscou - Leningrad, 1958, p. 422. 
trois classes du Registre du Millier. Il en va de même dans la liste des panetiers du rôle de la cour de $1546^{21}$.

Ces incohérences s'expliquent en grande partie par le fait que l'organisation territoriale de la cour subsiste encore au milieu du XVI ${ }^{\mathrm{e}}$ siècle : à l'intérieur de chaque classe, les membres du Millier étaient encore répartis selon les «villes » de province auxquelles ils étaient liés par le service et par la possession foncière. La sélection des « meilleurs serviteurs » s'opérait, selon toute vraisemblance, dans le cadre du district, en tenant compte de la hiérarchie de service propre à chaque ville. Cela n'entre pas en contradiction avec le fait que certaines villes n'ont pas donné de candidats pour les deux premières classes : l'analyse comparée du Registre $d u$ Millier et du Cahier de la cour montre que, dans ce cas, il ne se trouve pratiquement dans la ville en question aucun gentilhomme de lignage assez noble ou assez actif à la cour pour prétendre figurer dans les deux premières classes. Souvent, deux frères sont inscrits dans des classes différentes du Registre, comme le prince Dimitri Ivanovič Nemogo-Obolenskij, appartenant à la $1^{\text {re }}$ classe, tandis que son frère, le prince Théodore, figure dans la 3e. L'inclusion dans les classes supérieures ne dépendait pas seulement de l'ancienneté du lignage, mais aussi, dans une large mesure, des états de service de la personne. Ainsi, les princes Dimitri, Pierre et Ivan Kurakin, appartenant tous les trois à la première classe, sont mentionnés dans les registres des rangs à partir de 1538-1539, alors que leur frère cadet Grégoire, de la 2e classe, n'y apparaît qu'en 1556.

Il semble bien qu'on ait appliqué une règle générale, selon laquelle on ne pouvait accéder à l'une ou l'autre des deux premières classes qu'après être passé par la classe inférieure. Sur 54 membres du Millier présents à l'Assemblée des états en 1566 en qualité de gentilshommes de la première classe, la majorité (46) sont répertoriés dans la troisième par le Registre $d u$ Millier ${ }^{22}$. Par conséquent, malgré la prépondérance des aristocrates dans les classes supérieures du Registre, il serait faux d'affirmer que l'aristocratie s'opposait comme une caste à la masse des nobles ordinaires.

La réforme « du Millier » contribue à la réorganisation et à la restructuration de la cour. C'est ainsi que prend forme un nouveau groupe de serviteurs au sein de la cour - les gentilshommes choisis [sing. vybornyj dvorjanin], supérieurs à la masse des gentilshommes de la cour et des gentilshommes des villes en service ordinaire dans les corporations locales. Le rôle de la campagne de Polock de 1563 reflète parfaitement cette nouvelle structure de la cour. Voici comment il présente le régiment du tsar : «bojare, quartiers-maîtres, gens des bureaux, 41 personnes, panetiers, gentilshommes de la chambre, pages, 144 personnes, gentilshommes choisis, 374 personnes... $»^{23}$. Les membres de la cour (bojare, quartiers-maîtres, gens des bureaux (secrétaires), panetiers, gentilshommes de la chambre, gentilshommes

21. V.D. Nazarov, «O strukture... », op. cit. (note 8), p. 49-50.

22. A. A. Zimin, «Zemskij sobor 1566 g. » [ «L'assemblée des états de 1566 »], in IZ, vol. 71, Moscou, 1962, p. 205.

23. A. Sapunov, éd., Vitebskaja starina [Le Vitebsk d'antan], Vitebsk, 1885, vol. IV, p. 33. 
choisis) sont nettement distingués ici de la masse des gentilshommes de la cour et de province. Si les membres de la cour, y compris les gentilshommes choisis, constituent une sorte de corps d'officiers et sont nommément désignés à diverses fonctions, les gentilshommes de la cour, eux, comme les gentilshommes des villes, sont les combattants du rang qui composent l'armée. De sorte que dans les années 1550 et au début des années 1560 , les gentilshommes de la cour cessent d'être considérés comme membres de la cour et ne représentent plus qu'un grade au sein des corps nobiliaires dans les villes de province. Dès les années 1550, ils cessent pratiquement d'être convoqués pour servir à la cour, où ce ne sont plus désormais les gentilshommes de la cour, mais la nouvelle catégorie nobiliaire des gentilshommes choisis qui représentent les villes de province.

La réforme de la cour du milieu du $\mathrm{XVI}^{\mathrm{e}}$ siècle aboutit donc à une remise en ordre de ses effectifs comme de sa structure. Dans les années 1550-1560, on peut identifier les grades et groupes de grades suivants : grades du Conseil et officiers de la Couronne, panetiers, gentilshommes de la chambre, pages, gentilshommes choisis, et, probablement, secrétaires. Les nobles de la cour se distinguent de la masse des gentilshommes de province, dans la mesure où ils servent « de Moscou ».

Parallèlement à la consolidation de l'élite dirigeante, c'est-à-dire de la cour, le milieu du XVI ${ }^{\mathrm{e}}$ siècle voit la réorganisation des corps de serviteurs du prince dans les districts de province. Ils comprennent désormais deux grades, les « gentilshommes de la cour » [dvorovye deti bojarskie] et les « gentilshommes des villes » [gorodovye deti bojarskie]. Les membres de ces communautés nobiliaires sont désormais répertoriés dans des documents spéciaux, les dizaines [sing. desjatnja].

Cependant, la cour ne se sépare pas entièrement de la noblesse de province à l'issue de la réforme. C'est que, malgré les profonds changements intervenus, la structure de la cour continue à conjuguer deux principes fondamentaux d'organisation : le grade et l'appartenance territoriale. L'écrasante majorité des membres de la cour (à l'exception des bojare et des membres du Conseil, des principaux officiers de la Couronne et des secrétaires) appartiennent par ailleurs au corps nobiliaire de leur ville, qu'ils représentent en quelque sorte auprès du monarque. Dans les documents des années 1550-1560, on continue à les appeler d'après leur pays d'origine (gens de Kašira, de Kostroma, etc.) ${ }^{24}$. Le Registre du Millier de 1550 permet de constater que toutes les communautés nobiliaires locales un tant soit peu importantes comptent des représentants à la cour «réformée ». Qui plus est, le nombre de ces représentants de la noblesse provinciale est généralement proportionnel au nombre total de gentilshommes que compte chaque communauté de district. Si l'on confronte le Registre du Millier avec le rôle de la campagne de Polock (1563), on observe qu'à Vjaz'ma, sur 110 gentilshommes de la cour, 55 font partie du Millier ; à Dmitrov, 37 sur 110 ; à Dorogobuž, 9 sur 40 ; à Murom, 12 sur 40 , etc. ${ }^{25}$.

24. N. V. Mjatlev, Tysjačniki i moskovkoe dvorjanstvo XVI stoletija [Les bénéficiaires de la réforme du Millier et la noblesse moscovite au XVI siècle], Orel, 1912, p. 39-41.

25. A. P. Pavlov, Gosudarev dvor, op. cit. (note 15), p. 92-93. 
Même l'ancienneté du lignage est souvent sacrifiée, lors de la sélection du personnel de la cour, au souci d'assurer la représentation des communautés nobiliaires locales. Parmi les membres du Millier venus des villes de province (surtout des villes du Nord-Ouest et du Sud-Ouest), on rencontre souvent des rejetons de familles qui ne sont remarquables ni par la noblesse du lignage, ni par leurs états de service. Et cela alors qu'un certain nombre de princes de haute naissance, faisant partie du groupe des princes de Jaroslavl', de Rostov, etc., ne trouvent pas de place à la cour et doivent servir, à en juger par le rôle de la campagne de Polock, au milieu de simples gentilshommes de la cour ou des villes de province. Même le recrutement de cette élite de la cour qu'est le Conseil n'obéissait pas uniquement, comme l'ont fait remarquer Lev Čerepnin et Vladislav Nazarov, à la logique du lignage et du clan, mais aussi au principe territorial ${ }^{26}$.

En un sens, on peut dire que la cour du tsar qui prend forme en Russie entre la fin du XVe et le milieu du XVI ${ }^{\mathrm{e}}$ siècle est une sorte de « microcosme des communautés » nobiliaires locales (l'expression est de M. M. Bencianov, spécialiste de l'histoire de la cour et de la noblesse provinciale des $\mathrm{XV}^{\mathrm{e}}-\mathrm{XVI}$ siècles). Son recrutement est délibérément orienté en fonction d'un objectif précis : assurer la représentation des différentes communautés locales que l'on s'efforce d'impliquer dans le gouvernement de l'État ${ }^{27}$. Cette élite nobiliaire représentait les communautés locales non seulement à la cour, mais aussi auprès de l'Assemblée des états. Comme l'a souligné à juste titre Vladislav Nazarov, il serait historiquement erroné de ne voir dans ces gentilshommes de la cour qui prennent part aux Assemblées des états dans les années 1550-1560, alors que la cour est encore organisée selon le principe territorial, que des officiers du monarque auxquels on a imposé cette mission ${ }^{28}$. L'ordre dans lequel sont disposés les noms des nobles appartenant à la première et à la deuxième classe du Registre du Millier qui ont participé à l'Assemblée de 1566 ne doit rien au hasard. Il correspond globalement, comme l'a montré Aleksandr Zimin, aux différentes communautés locales dont ils faisaient partie ${ }^{29}$. Dans les listes de nobles ayant participé à l'Assemblée, on peut identifier des groupes assez compacts d'habitants de Vjaz'ma, de Galič, de Novgorod, etc., bien que dans la charte approuvée par l'Assemblée de 1566, à la différence du Registre du Millier et du Cahier de la cour, ces groupes territoriaux ne soient pas classés sous des rubriques séparées. La pratique de l'élection sur place des gentilshommes délégués aux Assemblées d'états ne se met en place définitivement qu'à la fin du XVI siècle,

26. Voir L. V. Čerepnin, Zemskie sobory Russkogo gosudarstva XVI-XVII VV. [Les Assemblées des états en Russie aux XVI $-X V I I^{e}$ siècles], Moscou, 1978, p. 5-47; V. D. Nazarov, «Gosudarev dvor i soslovnoe predstavitel’stvo na Rusi v XVI v. (tradicii i izmenenija) » [« La cour du tsar et la représentation des états en Russie au XVI ${ }^{\mathrm{e}}$ siècle (tradition et changements) »], in Obščestvo, gosudarstvo, pravo Rossii i drugih stran Evropy [La société, l'État, le droit en Russie et dans d'autres pays européens], Moscou, 1983, p. 60.

27. M. M. Bencianov, « Gosudarev dvor i territorial'nye korporacii služilyh ljudej russkogo gosudarstva v konce XV - načale XVI v. » [La cour du tsar et les corporations territoriales des serviteurs du prince en Russie à la fin du XVe - début du XVI e siècle], résumé de thèse, Ekaterinburg, 2000, p. 3.

28. D. N. Nazarov, « Gosudarev dvor... », op. cit. (note 26), p. 60.

29. A. A. Zimin, « Zemskij sobor», op. cit. (note 22), p. 205. 
lorsque la structure des grades de la cour est définitivement adoptée et que les grades moscovites sont nettement séparés des grades provinciaux ${ }^{30}$.

Les réformes du milieu du XVI $\mathrm{XI}^{\mathrm{e}}$ siècle ont eu pour effet d'estomper les contradictions entre les différentes couches nobiliaires, la cour est apparue comme l'une des composantes de l'ordre de la noblesse. La petite noblesse de province obtint de cette manière des droits et des privilèges jadis réservés aux seuls membres de l'élite. Ainsi, l'édit de 1549 (dont les dispositions furent ensuite confirmées par le Justicier de 1550) dispensa les gentilshommes de province du tribunal du bailli [namestnik] (à l'exception des crimes les plus graves) et les autorisa à recourir aux tribunaux du pouvoir central. C'était un pas en avant considérable vers la création d'un tribunal spécifique de la noblesse et vers l'unification de celle-ci. Par ailleurs, l'Établissement [Uloženie] de 1556 concernant le service militaire adopte comme base de calcul une même superficie (100 arpents, soit environ 150 hectares), dont le possesseur, qu'il s'agisse d'un bénéfice ou d'un patrimoine, doit fournir un cavalier armé, ce qui revient à égaliser pratiquement le service exigible de ces deux types de possession foncière. Cette législation achève le processus de formation d'un ordre unique des serviteurs militaires, dont tous les membres avaient l'obligation de servir le tsar. Désormais, ni les membres de la cour dans leur ensemble, ni même l'aristocratie qui en formait le sommet ne constituaient une oligarchie de magnats, de grands propriétaires fonciers tout-puissants dans leur province ; ils n'étaient que l'élite dirigeante des serviteurs du tsar.

Cette évolution de l'élite dirigeante s'inscrit bien dans la logique générale des réformes du milieu du $\mathrm{XVI}^{\mathrm{e}}$ siècle, qui ont clairement pour but la consolidation des différents ordres [sing. soslovie] en Russie, l'élargissement de leurs droits corporatifs et le développement de leur représentation. Les nobles du rang obtiennent du gouvernement des concessions significatives, tant politiques qu'économiques. Outre la création d'une justice unique pour tous les membres de la noblesse, on peut citer l'interdiction légale faite aux grands seigneurs de réduire en servitude [obraščat' $v$ holopy] les gentilshommes aptes au service. Pour satisfaire les exigences de vastes couches de la noblesse, qui réclamaient une répartition équitable des impôts, le Justicier de 1550 promulgue (article 43) l'annulation des immunités fiscales [sing. tarhan] dont jouissaient les grands propriétaires ecclésiastiques. Les intérêts des citadins sont également protégés. L'article 91 du Justicier interdit aux membres des communautés urbaines d'élire domicile dans les parcelles privilégiées du territoire urbain appartenant aux grands seigneurs. C'est la reconnaissance de facto par le gouvernement du monopole des citadins sur le commerce et l'artisanat urbains.

Mais la concession de loin la plus importante du gouvernement consentie aux ordres en voie de formation fut l'octroi d'une large autonomie, conséquence de la réforme de l'administration locale.

Déjà, à la fin des années 1530 , commence la mise en place de la réforme judiciaire [gubnaja reforma], qui prévoit de retirer aux baillis les affaires criminelles pour les remettre aux officiers élus de l'administration locale de la justice et de la 
police [gubnye organy], chargés désormais de rechercher et d'appréhender les brigands dans le cadre de leur ressort. Ces offices sont le premier embryon d'une auto-administration locale de l'ordre de la noblesse et représentent un progrès considérable dans la consolidation de celle-ci ${ }^{31}$.

L'évolution ultérieure du système des institutions électives d'auto-administration locale coïncide avec les réformes du milieu du XVI siècle. L'événement majeur de la période fut l'abolition en 1555/56 de l'ancien système d' " assignation en subsistance » [sing. kormlenie], ce qui réduisit le rôle des élites moscovites dans l'administration locale. Toutefois, contrairement à l'opinion reçue, cette mesure ne visait pas les bojare. Les princes et les bojare n'avaient pas le monopole des charges de bailli et de viguier [volostel'], qui ouvraient droit à l'assignation en subsistance. Ce privilège s'étendait à un cercle assez large de serviteurs du tsar (plusieurs centaines d'individus), principalement des membres de la cour. Pour compenser la perte des assignations en subsistance, on leva sur la population un nouvel impôt, «l'argent des subsistances », dont le produit était ensuite réparti entre les serviteurs du tsar qui avaient jadis droit à l'assignation en subsistance. L'abolition des assignations en subsistance, la rémunération des serviteurs sous forme de gages [žalovanie] en argent payés par l'État représente un pas de plus, et un pas important, vers la formation d'un ordre unique des serviteurs militaires de la monarchie.

Une des conséquences de l'abolition des assignations en subsistance fut de faire passer le pouvoir local entre les mains des officiers élus. La réforme judiciaire est achevée vers le milieu des années 1550. L'administration locale de la justice et de la police se développe dans l'ensemble du pays. La compétence des lieutenants criminels [sing. gubnyj starosta] s'étend désormais non seulement aux affaires de brigandage, mais aussi aux questions d'administration locale les plus diverses, y compris celles qui ont trait à la propriété foncière. Gentilshommes élus par leurs pairs, les lieutenants criminels s'inspirent, dans l'exercice de leur charge, des intérêts de l'ordre auquel ils appartiennent. Grâce à eux, la noblesse provinciale est désormais capable de défendre elle-même ses intérêts.

La noblesse n'est pas la seule à obtenir, au milieu du XVI e siècle, le droit de s'auto-administrer et d'exercer un pouvoir local réel. La réforme de l'administration locale [zemskaja reforma] de 1551-1556 revêt également une grande importance sociale et politique ${ }^{32}$ : là où il n'y avait pas de propriété seigneuriale, le pouvoir passait entre les mains des syndics [sing. starosta] et de leurs adjoints, jurés [sing. celoval'nik] et secrétaires [sing. d’jak] des communautés locales, élus par les paysans et les citadins. Les syndics, élus le plus souvent parmi les paysans et les citadins les plus aisés, défendaient activement les intérêts locaux du tiers-état en devenir. À la faveur de la réforme de l'Église, comme l'a montré Boris Nikolaevič

31. Voir N. E. Nosov, Očerki po istorii mestnogo upravlenija Russkogo gosudarstva pervoj poloviny XVI V. [Essais sur l'histoire de l'administration locale en Russie dans la première moitié du XVI siècle], Moscou — Leningrad, 1957.

32. N. E. Nosov, Stanovlenie soslovno-predstavitel’nyh učreždenij v Rossii : izyskanija o zemskoj reforme Ivana Groznogo [La genèse des institutions représentatives des ordres en Russie : études sur la réforme d'Ivan le Terrible], Leningrad, 1969. 
Florja, le clergé paroissial se voit doter également d'une certaine autonomie, par l'intermédiaire de «doyens du clergé » [sing. popovskij starosta] élus, et d'une indépendance relative à l'égard du pouvoir laïc ${ }^{33}$.

On peut en conclure que les réformes du milieu du XVI $\mathrm{I}^{\mathrm{e}}$ siècle ont clairement tendu à favoriser le développement des droits corporatifs et l'auto-administration des différents ordres, touchant ainsi les couches les plus diverses de la société russe : la noblesse, le clergé, la paysannerie et la population citadine.

Comme nous l'avons déjà constaté en analysant la réforme de la cour, le gouvernement n'oublie pas pour autant de sauvegarder les droits et privilèges de l'aristocratie et des couches supérieures de la noblesse, c'est-à-dire de l'élite dirigeante de la société russe. Les réformes du milieu du XVI siècle ne purent du reste être mises en œuvre que grâce à la consolidation de ces élites, et avant tout de l'aristocratie des bojare. Contrairement à une opinion répandue, non seulement l'aristocratie ne s'est pas opposée à la centralisation du pays, mais elle avait intérêt à conserver un pouvoir monarchique fort, capable de garantir la pérennité de la hiérarchie traditionnelle du service et des préséances, hiérarchie grâce à laquelle surtout elle parvenait à se maintenir au sommet de la pyramide sociale. De larges mouvements populaires (avant tout, l'insurrection de Moscou de 1547) dirigés contre les bojare favoris du prince avaient obligé l'aristocratie à réfléchir sérieusement à son avenir. Le désir de conserver leur situation momentanément compromise, leur influence de jadis et leur autorité dans le pays poussait les membres de l'aristocratie à se souder autour du trône. Les recherches récentes soulignent le rôle actif joué dans la mise en œuvre des réformes par les aristocrates, aussi bien les rejetons de maisons princières que les vieilles lignées de bojare (non titrés) de la principauté de $\mathrm{Moscou}^{34}$. Ce n'est pas un hasard si parmi les toutes premières mesures du gouvernement des réformateurs du milieu du $\mathrm{XVI}^{\mathrm{e}}$ siècle figurent des mesures tendant à renforcer l'importance du Conseil, organisme représentatif de l'aristocratie. On lit par exemple dans le Justicier de 1550, article 98, que toutes les nouvelles lois promulguées après l'adoption du Justicier devaient être rapportées au souverain par le Conseil et confirmées par celui-ci ( Si des cas nouveaux se présentent, qui ne sont pas traités dans le présent Justicier, lorsqu'ils auront été résolus après rapport au souverain et arrêt de tous les bojare, qu'ils soient ajoutés dans le Justicier » ${ }^{35}$ ). Le Conseil ne limitait en rien le pouvoir du tsar (et ne cherchait d'ailleurs pas à le faire) ; ses fonctions étaient loin d'être purement décoratives pour autant. Plus l'appareil administratif de l'État (les secrétariats [sing. prikaz]) croissait et se perfectionnait, plus le rôle du Conseil devenait considérable, car c'était lui qui coordonnait la marche de cet appareil ${ }^{36}$. L'article 98 du Justicier de 1550 ne fait que donner une forme légale à des pratiques qui remontent à la première moitié du

33. B. N. Florja, Otnošenija gosudarstva i cerkvi u vostočnyh i zapadnyh slavjan [Les rapports de l'État avec l'Église chez les Slaves orientaux et occidentaux], Moscou, 1992, p. 78-80.

34. B. N. Florja, Ivan Groznyj [Ivan le Terrible], Moscou, 1999, p. 50.

35. Sudebniki, $X V$-XVIVV. [Les Justiciers, $X V^{e}-X V I^{\mathrm{e}}$ siècles], Moscou-Leningrad, 1952, p. 176.

36. A. P. Pavlov, Gosudarev dvor, op. cit. (note 15), p. 228-232. 
siècle et qui font du Conseil l'organe gouvernemental suprême auprès du monarque.

Ainsi, les réformes du milieu du XVI ${ }^{\mathrm{e}}$ siècle, en visant une sorte de compromis entre les intérêts de l'État et ceux des ordres en voie de formation, ont réussi à satisfaire les intérêts des groupes sociaux les plus divers, la noblesse provinciale, les citadins, l'aristocratie des bojare.

Il est évident que le degré de développement des ordres en Russie était embryonnaire, comparé aux pays d'Europe occidentale où pourtant ils étaient encore en voie de formation. Ce développement était, de plus, rendu difficile par l'intervention de l'État et par ses tentatives de soumettre les institutions locales autonomes au contrôle du pouvoir central. Le travail des lieutenants criminels était supervisé depuis Moscou par le Secrétariat au brigandage, tandis que le fonctionnement de l'auto-administration locale était bridé par le principe de responsabilité collective [krugovaja poruka]. L'évolution vers une administration locale gérée par la noblesse allait de pair avec l'accroissement du fardeau fiscal. Pourtant, dans un climat propice à l'élargissement des droits corporatifs, à l'administration des différents ordres par eux-mêmes (pratiquée du reste à des niveaux très variables), l'engagement de la Russie dans la voie de développement suivie par les pays d'Europe occidentale était parfaitement pensable. Cette voie aurait pu aboutir graduellement à un régime où la représentation des ordres aurait limité le pouvoir du souverain. Nikolaj Evgen'evič Nosov fut le premier à observer cette tendance et à présenter des arguments tout à fait convaincants dans ce sens ${ }^{37}$.

Parmi les résultats les plus importants des réformes du milieu du XVI $\mathrm{X}^{\mathrm{e}}$ siècle, il faut compter la formation d'un ordre unique de la noblesse, à fonction militaire, dont tous les membres (du bojarin aristocrate au gentilhomme provincial du rang) étaient unis par des droits et des obligations communs : obligation de servir le souverain, droit d'obtenir, en échange de ce service, des bénéfices et des gages en argent, droit d'être justiciable directement du monarque et non des autorités locales. L'ancienne élite dirigeante, à qui l'unification et la centralisation de l'État avaient fait perdre une partie de ses privilèges (le droit de désaveu, l'immunité fiscale, etc.), retrouvait, comme l'a fait remarquer Boris Nikolaevič Florja, un poids accru et toute son influence, cette fois en tant que couche supérieure de la nouvelle noblesse. Les communautés nobiliaires locales gagnant en importance, l'aristocratie aurait pu, en s'appuyant sur elles et en défendant leurs intérêts à la cour, acquérir une plus grande indépendance à l'égard de la monarchie et imposer des limites au pouvoir autocratique du souverain ${ }^{38}$.

Mais cette tendance de l'évolution ne devait pas aboutir. L'opričnina, ou domaine réservé, d'Ivan IV provoqua de profonds changements dans la vie des différents ordres, surtout dans celle de la noblesse, changements qui allaient peser par la suite sur le développement de l'État en Russie. Les transferts fonciers opérés dans le cadre de l'opričnina eurent pour effet de couper une grande partie de la

37. N. E. Nosov, Stanovlenie, op. cit. (note 32), p. 9-13.

38. B. N. Florja, Ivan Groznyj, op. cit. (note 34), p. 54-55. 
noblesse russe des régions où elle s'était acclimatée ${ }^{39}$. Les liens traditionnels de service ou d'intérêts fonciers qui unissaient la noblesse furent sérieusement compromis, ce qui affaiblit les communautés nobiliaires locales face à la monarchie. L'opričnina porta un rude coup aux patrimoines ancestraux des bojare et plus particulièrement aux patrimoines princiers. À la fin $\mathrm{du} \mathrm{XVI}^{\mathrm{e}}$ siècle, la plupart des rejetons de familles princières avaient perdu leurs terres patrimoniales héréditaires. La ruine des domaines patrimoniaux de l'aristocratie, la rupture des liens que celleci entretenait avec la noblesse de province, la coupure entre nobles de l'opričnina et nobles « du pays » [zemskie] amenèrent une profonde transformation de l'aristocratie russe, la rendant entièrement dépendante de la monarchie. La destruction des relations existantes entre propriétaires fonciers modifia la structure même de la noblesse : l'appartenance territoriale comme principe d'organisation de la cour fut abandonnée au profit de la classification par grades. Une nouvelle élite apparaît, se détachant de la masse des simples gentilshommes : il s'agit des grades de Moscou (panetiers, gentilshommes de la chambre, gentilshommes de Moscou). Ils sont totalement coupés de la noblesse de province, et effectuent leur service exclusivement « selon le rôle de Moscou », ce qui accroît leur dépendance à l'égard du pouvoir de l'État ${ }^{40}$. Pendant et après l'opričnina, le processus de consolidation de l'élite nobiliaire, c'est-à-dire de la cour, se poursuit, mais sur des bases tout à fait différentes de ce qu'elles étaient au milieu du XVI siècle. L'élite de la cour (membres du Conseil, grades de Moscou, secrétaires) se trouve opposée à la masse des nobles de province, qui sont de fait écartés des hautes sphères du pouvoir. Globalement, la noblesse russe évolue non pas vers plus de cohésion, mais vers la division en grades, l'aristocratie privilégiée s'opposant aux sans-droits de la petite noblesse. La noblesse, incapable de défendre ses intérêts politiques, perd en conséquence de cette métamorphose non seulement sa capacité à s'opposer à la monarchie, mais son existence même en tant qu'ordre nobiliaire. Ces facteurs réunis ont permis au pouvoir, dans le dernier tiers du XVI ${ }^{\mathrm{e}}$ siècle, de s'élever au-dessus de la société et d'adopter définitivement la voie de l'autocratie.

(traduit du russe par André Filler et André Berelowitch)

Académie des sciences de Russie

Institut d'histoire de la Russie

Saint-Pétersbourg

pavlov_ap@mail.ru

39. A. P. Pavlov, «Zemel’́nye pereselenija v gody opričniny »[« Transferts fonciers des années de l'opričnina »], Istorija SSSR (Histoire de 1'URSS), n 5, 1990, p. 89-104. Sur les déportations massives des propriétaires locaux, voir également A. V. Antonov, K. V. Baranov, éds., Akty služilyh zemlevladel'cev, 1400-1650 [Archives des propriétaires fonciers au service du prince, 1400-1650], Moscou, 1997-2002, vol. 1-3.

40. A. P. Pavlov, Gosudarev dvor, op. cit. (note 15), p. 100-103, 151-160. 